IZA DP No. 8710

Pour Some Sugar in Me:

Does Glucose Enrichment Improve Decision Making?

Todd McElroy

David L. Dickinson

Nathan Stroh

December 2014 


\title{
Pour Some Sugar in Me: Does Glucose Enrichment Improve Decision Making?
}

\author{
Todd McElroy \\ Florida Gulf Coast University
}

David L. Dickinson

Appalachian State University

and IZA

Nathan Stroh

Appalachian State University

\section{Discussion Paper No. 8710 \\ December 2014}

\author{
IZA \\ P.O. Box 7240 \\ 53072 Bonn \\ Germany \\ Phone: +49-228-3894-0 \\ Fax: +49-228-3894-180 \\ E-mail: iza@iza.org
}

Any opinions expressed here are those of the author(s) and not those of IZA. Research published in this series may include views on policy, but the institute itself takes no institutional policy positions. The IZA research network is committed to the IZA Guiding Principles of Research Integrity.

The Institute for the Study of Labor (IZA) in Bonn is a local and virtual international research center and a place of communication between science, politics and business. IZA is an independent nonprofit organization supported by Deutsche Post Foundation. The center is associated with the University of Bonn and offers a stimulating research environment through its international network, workshops and conferences, data service, project support, research visits and doctoral program. IZA engages in (i) original and internationally competitive research in all fields of labor economics, (ii) development of policy concepts, and (iii) dissemination of research results and concepts to the interested public.

IZA Discussion Papers often represent preliminary work and are circulated to encourage discussion. Citation of such a paper should account for its provisional character. A revised version may be available directly from the author. 
IZA Discussion Paper No. 8710

December 2014

\section{ABSTRACT \\ Pour Some Sugar in Me: \\ Does Glucose Enrichment Improve Decision Making?}

In the current study we explore whether enriching the brain's supply of glucose will improve the quality and speed of decision making. Prior research shows that glucose enrichment supports cognition and more recent research has shown it can improve decision making on some tasks. To test our hypothesis we used a standardized decision inventory and measured response times. The findings show that supplemental glucose improves decision making but only in complex decision tasks. The findings also show that enrichment leads to faster decision response times across decision types.

\section{NON-TECHNICAL SUMMARY}

We show that glucose supplementation improves outcomes in a standardized complex decision making inventory. Glucose supplementation also increases response times across all decision domains in the inventory. Given the frequent use of sugary drinks or snacks in modern society, to include the workplace, these results suggest at least one possible benefit of elevated glucose levels for decision making. Implications may also extend to those with hypo- or hyperglycemic conditions. Finally decision making over the course of the day may be predictably impacted due to natural variations in blood glucose levels.

JEL Classification: $\quad$ C91

Keywords: $\quad$ glucose, response time, A-DMC, thinking, experiments

Corresponding author:

Todd McElroy

Department of Psychology

Florida Gulf Coast University

10501 FGCU Blvd, South

Fort Myers, FL 33965-6565

USA

E-mail: tmcelroy@fgcu.edu 
As research has shown, many factors may influence how much we think about a decision; importance, relevance, and even time of day can affect the degree to which we thoughtfully consider a decision. In the current study we investigate how the brain's fuel source for thinking, glucose, improves the quality and speed of decision making.

\section{Glucose and Cognition:}

The human brain is small when compared to overall body mass, representing only about $2 \%$ of the total weight of an adult human, yet it utilizes $20-30 \%$ of the body's total energy needs (Benton, 1990). Glucose fuels this massive energy consumer almost exclusively, making it a key variable for autonomic and executive brain functions.

Glucose, simply put, is sugar present in the bloodstream. The level of blood glucose varies considerably because the brain is surprisingly poor at storing it for extended periods and it requires a continuous supply (Bos, Dijksterhuis, \& can Baaren, 2012; Benton, 1990; GonderFrederick, et al. 1987). Following the consumption of food or drink containing sugar, blood glucose levels normally rise sharply after about 12-15 minutes and return to baseline over the course of about 2 hours. If a shortage of glucose occurs, then the brain cannot function optimally and a variety of cognitive functions will likely be affected.

As evidence to this, glucose demand and usage appear to parallel cognitive thought. This relationship is highlighted in a study by Donohoe and Benton (1999) wherein the researchers used PET scans to observe participants who first consumed a glucose or placebo drink. After consuming the drink, participants performed a cognitively demanding rapid visual information task or they sat in a control room. The PET scans revealed that participants performing the cognitively demanding visual task had significantly lower glucose levels, indicating a greater usage of blood glucose during the task. 
Researchers have also looked at how glucose levels influence a variety of tasks that draw upon more specific cognitive processes. For example, when glucose deprived participants are given a glucose enriching substance, research has shown performance improvements in facial recognition tasks (Metzger, 2000), verbal working memory (Messier, Pierre, Desrochers \& Gravel, 1998; Sunram-Lea, Foster, Durlach \& Perez, 2001; Sunram-Lea, Foster, Durlach \& Perez, 2002) and spatial abilities (Sunram-Lea, Foster, Durlach \& Perez, 2001). In a similar manner, glucose deprivation has been shown to inhibit performance in several complex tasks such as mental calculation (Schächinger, Cox, Linder, Brody, \& Keller, 2003) and the Stroop task (Benton, Owens \& Parker, 1994).

While there is good evidence that cognition depends upon glucose, there is also evidence that the magnitude of glucose's influence may vary with the complexity of the task. For example, in a study by Kennedy \& Scholey (2000) the researchers tested glucose effects on tasks varying in complexity including a Serial Threes, Serial Sevens and Word Retrieval task. Their overall findings showed that glucose improved performance but only on the most complex task. Perhaps the best evidence that cognitively complex tasks are more heavily dependent upon glucose can be found in a study by Scholey, Harper, and Kennedy (2001). In this study, Scholey et al. (2001) included a balanced design, controlling for domain (word tasks) and cognitive demand while manipulating glucose deprivation and measuring blood-glucose levels. Their findings again showed that glucose improved performance on the more cognitively demanding task but not on the simple task. Altogether these studies provide compelling evidence that complex tasks are more dependent upon and affected by glucose level.

A related line of research has also shown that blood glucose levels influence the speed of cognitive processing, such that, enriched blood glucose levels are associated with faster cognitive 
processing and shorter response times (Owens, \& Benton, 1994). For example, in one study Benton, Owens and Parker (1994) (See also; Benton \& Nabb, 2003) administered several tasks, one of which was the Rapid Information Processing Task which is designed to measure processing speed. They found that a glucose drink improved reaction times on this task. Owens and Benton concluded that increasing levels of blood glucose seemed to speed up cognitive processing and, in turn, lead to the faster reaction times observed in the Information Processing Task. Similar results were found in a Bayes updating task (Dickinson, McElroy \& Stroh, in press).

\section{Glucose effects on decision making:}

Recently, researchers have begun to examine the role of glucose in different types of decision tasks. In one study McMahon and Scheel (2010) focused on decisions involving probability learning. They found that when transitioning from the more simple maximization strategy to the more thoughtful rule-based probability matching one, participants in the glucosedeprived condition engaged in more simple maximization strategies. Participants in the glucoseenriched condition were more likely to follow a rule-based probability approach. This finding suggests that a lack of glucose leads to reliance on decision strategies that are less effortful.

Masicampo and Baumeister (2008) used a different type of decision task to test glucose effects on decision making. In this study they used an attraction task wherein participants first evaluate two options based on different attributes. A third "decoy" option, which is inferior on all attributes, acts to lead decision makers toward whichever alternative is more similar to the decoy. Prior research shows that reliance on the decoy option reflects more heuristic, less effortful decision making (Simonson, 1989). Masicampo and Baumeister report that glucose deficient participants were more likely to make less optimal choices by relying on the decoy, 
which again suggests more heuristic, less effortful decision strategies for glucose deficient individuals.

In another investigation, Wang and Dvorak (2010) looked at how glucose influences future discounting, a phenomenon wherein future rewards are seen as less valuable than immediate rewards. Their findings showed that glucose deprived participants were more likely to engage in future discounting whereas enriched participants were better able to regulate the value of expected future rewards versus immediate payoffs in a decision.

Together these studies suggest that glucose deprivation is associated with less thoughtful decision making and more reliance on simple decision strategies whereas glucose enrichment leads to more deliberative, thoughtful decisions. However, it is unclear whether glucose improves decision making across all types of decisions or whether it is limited to more complex decisions as other research suggests (e.g., Scholey et al., 2001). Further, with few exceptions (e.g., Dickinson, McElroy, Stroh, in press) research has not examined whether glucose facilitates the speed of decision making as it does in some types of cognitive tasks (e.g., Benton et al., 1994). The present study was designed to examine these questions.

\section{Summary and Predictions:}

Overall, the research investigating how glucose affects cognitive functioning has revealed that depleting the brain's fuel source can impede cognitive performance whereas enhancing glucose appears to improve it. Research in decision making suggests similar effects, reliance on simpler decision strategies under glucose deprivation and more thoughtful strategies under conditions of glucose enrichment. This effect seems to be more robust in cognitively complex tasks and less so in simpler tasks. Research has also shown that the brain's processing speed is impeded with glucose deprivation and facilitated with glucose enrichment. 
Based upon the findings from previous research we developed hypotheses for how glucose should affect decision making.

Hypothesis 1: Glucose enriched participants will have improved decision making performance relative to glucose depleted participants and this difference will be most pronounced for complex decision tasks.

Hypothesis 2: Glucose enrichment will facilitate processing speed. Because faster processing speed should lead to quicker response times, glucose enriched participants should have decreased response times.

\section{Method}

In the current investigation we set out to study the effects of glucose on decision making. We adopted a standardized method using a sugar drink and a placebo to manipulate glucose. Because we wanted to observe a broad spectrum of decision making, we decided to utilize the Adult Decision Making Competence Scale (A-DMC) (Bruine de Bruin, Parker, \& Fischhoff, 2007) a robust and well validated measure of decision making. While this task was comprehensive, it was also a paper-and-pencil task. Because a foremost concern from our reading of the literature was that glucose had pronounced effects on processing speed, we programmed the A-DMC into software that would allow us to precisely measure participant's response time speed along with decision choice for each of the decision tasks.

\section{Participants and Design}

One hundred and thirty eight glucose deprived participants (Fasting for $>3$ hours) (98 females) took part in the study. Participants were all undergraduates and were recruited using the Sona software system. Participants received credits toward fulfilling a requirement for an undergraduate psychology course. The design of the study included the independent variable of 
glucose level (enriched or deprived), which was manipulated via random assignment of either regular (sugar sweetened) lemonade (40 grams sugar) or sugar-free lemonade (placebo, 0 grams sugar). The dependent variables were aggregate scores and average response times derived from the A-DMC.

\section{Procedure}

When participants initially signed up for the study they were provided with basic information including the need to fast for at least three hours before the study began. Participants who had glucose sensitivity were asked not to sign up for the study. The minimum amount of time allowed between study signup and the study start time was 24 hours so that participants could prepare for fasting. The evening before the study was to take place, participants were emailed and reminded again not to eat or drink anything for at least three hours before their study session was set to begin. Study sessions took place during the morning hours to help participant's comply with the fasting requirement during their academic day. Thus, all of the participants who followed instructions should have arrived in a glucose-deprived state.

Each study session included one to three participants. Participants were seated at an individual study carrel that contained a standard computer setup with monitor and keyboard. Upon arrival, participants were first provided with informed consent ${ }^{1}$. After consent was obtained, participants were instructed to drink the lemonade that had been placed on the study carrel in front of them. After completing consumption of the lemonade drink, participants were presented with several unrelated filler tasks that had been devised to take approximately fifteen minutes. The purpose of these filler tasks was to provide sufficient time for the glucose to be absorbed into the bloodstream (e.g., Masicampo \& Baumeister, 2008). After completing the

\footnotetext{
${ }^{1}$ Because of the double-blind procedure, one participant was granted credit and dismissed from the study due to concerns about the contents of the drink.
} 
filler tasks, participants were given instructions to begin the computerized A-DMC task that was presented in E-Prime software. Any remaining instructions were provided within the computerized version of the task. After completing the A-DMC, participants were instructed to wait quietly until everyone had finished the task. Participants were then debriefed about the study, provided with another opportunity to ask any questions, and thanked for their participation.

\section{Materials}

Glucose manipulation. To manipulate glucose level we used a procedure conceptually similar to prior experiments investigating glucose levels (e.g., Masicampo \& Baumeister, 2008; McMahon \& Scheel, 2010). In this procedure a sugar drink or placebo is consumed and a distractor task takes place for 10-15 minutes giving the sugar time to be absorbed into the bloodstream. Well in advance of our study, a research assistant who did not act as experimenter prepared the drink manipulation. This preparation consisted of covering the drink can with a gray foam cover and black electrical tape so that no part of the can's label could be seen by participants. The drink was then coded with a subject number. The condition (glucose or placebo) was recorded separately and stored in a password-protected spreadsheet not accessible to the experimenter. This double-blind procedure allowed us to be confident that neither the participant nor the experimenter was aware of any individual participants' assigned condition.

To manipulate glucose we used a Minute Maid ${ }^{\circledR}$ Lemonade drink that can be purchased at most grocery stores. We chose to use this standard drink because it is something that participants would commonly experience and pretesting indicated that the sugar-free lemonade drink tasted very similar to the regular lemonade. Both drinks were in $12 \mathrm{oz}$. cans. The regular Lemonade contained $40 \mathrm{~g}$ of sugar and the Light Minute Maid Lemonade contained $0 \mathrm{~g}$ of sugar. 
In an attempt to maintain consistency in time of consumption, participants were instructed to drink the lemonade as quickly as possible.

The A-DMC. The Adult Decision Making Competence Inventory (A-DMC) (Bruine de Bruin et al., 2007) is a well validated and reliable measure of competency in decision making and is based upon prior achievements of DMC development (e.g., Parker, \& Fischhoff, 2005). The inventory consists of 134 individual items and contains six subsections. The decision making ability measured by the A-DMC should be considered a trait that varies with respect to the individual (e.g., Stanovich \& West, 2000). In support of this, individuals who score higher on the A-DMC report having fewer negative decision outcomes in their lives; they also tend to have higher education levels and greater cognitive ability (Bruine de Bruin et al., 2007). Prior research has presented the A-DMC in a paper and pencil format. However, because one of our hypotheses centered on the role of glucose in processing speed, we programmed the A-DMC into Eprime software to measure participant's response times. All task information was consistent with the original version and the integrity of the images was maintained using Photoshop ${ }^{\circledR}$.

Resistance to Framing. Resistance to Framing reflects the extent to which variations in how the problem is presented or framed influence decision choice. Because framing effects represent a form of decision bias, resistance to these effects has been taken as a positive indicator of decision making competency (DMC). Resistance to Framing is composed of two different types of framing tasks; risky choice and attribute (Levin, Schneider \& Gaeth, 1998). These two types of framing are measured by seven problems each, with each problem being presented in both a positive and negative frame. Importantly, each type of framing is manipulated within-subjects. Both the positive and negative versions of the task are spaced well apart, appearing after a number of intervening tasks, so as to minimize the chance that 
participants simply remember and repeat an earlier response when they receive the second version of the same problem. Thus, this subsection represents a balance between risky choice and attribute framing. In the risky choice problems participants are presented with a situation (e.g., the outbreak of a disease) followed by both a sure option and a risky option of equal expected value. The options are framed either positively (people saved) or negatively (people die). In the attribute framing problems participants are presented with normatively equivalent events (e.g., buying ground beef) wherein the key attribute is described in either positive (80\% lean) or negative (20\% fat) terms. A 6-point scale was used for rating both types of framing tasks, this allows for assessment of even weak preferences toward an alternative (Levin, Gaeth, Schreiber, \& Lauriola, 2002). The tasks are scored using the mean absolute difference between ratings for the loss and gain versions so that higher scores represent greater framing effects. Later we describe how we use the combined measure and then a separate measure to test attribute and risky choice framing effects.

Recognizing Social Norms. Recognizing Social Norms is a measure of an individual's ability to assess social appropriateness of certain norms and their propensity to engage in these peer related social interactions. In this task participants are presented with 16 different negative behaviors (e.g., Do you think it is sometimes OK to steal under certain circumstances?). They are asked to initially rate the acceptability of the bad behavior and later they are asked to estimate the percentage of people who would support this negative behavior. Performance is measured by the strength of the relationship between acceptability of the behavior and estimated percentage of peer endorsements of the interactions.

Under/overconfidence. Under/overconfidence is a measure of how well calibrated individuals are at assessing the correctness, or accuracy, of their responses. In this section 
participants are first presented with 34 statements (e.g., Amman is the capital of Jordan.) and asked to indicate whether they believe these statements to be true or false. Next, participants are asked to rate on a $50 \%$ to $100 \%$ scale how confident they are in their true or false assessment. For example, after answering true or false to the statement "alcohol causes dehydration," participants then rate their confidence in that answer. Performance is assessed by calculating the absolute difference between mean confidence and percentage correct and higher scores reflect smaller differences between confidence and correct responses which is indicative of better calibration.

Applying Decision Rules. The decision rules task in the A-DMC was purposely redesigned to be more complex than decision rules sections in previous DMC versions. This subsection involves having individuals use different decision rules to indicate which of five DVD players they would purchase in a hypothetical situation. Participants are first provided with a hypothetical persons' decision rule (e.g., Brian selects the DVD player with the highest number of ratings greater than "Medium".) and then asked to make a choice among five DVD players. Aspects of the DVD players such as sound quality and brand reliability vary on a fivepoint scale. Participants' performance is assessed by the percentage of correct DVD players chosen, given the decision rules that should be applied.

Consistency in Risk Perception. Risk perception is a measure of a participant's ability to follow probability rules. Participants are asked to rate the likelihood of a given event happening to them (e.g., "what is the probability that you will have a cavity filled during the next year?”). The probability rating ranges from $0 \%$ to $100 \%$ and the probability of each event is assessed for the "next year" and "the next 5 years" in separate parts of the survey. Each time the frame pair is scored as correct if the probability for the event happening the next year is less than or equal to 
it happening in the next 5 years. Within each time frame, three item pairs are presented as nested subset and superset events. In order to be accurate, the probability of a subset event cannot exceed a superset event. Additionally, within each time frame two complementary events are presented, as such their combined probability must total $100 \%$ to be scored as correct.

Resistance to Sunk Costs. Sunk cost is a measure of participants' ability to avoid the entrapment of prior investments in a particular target item. In this task participants are presented with ten scenarios (e.g., You are buying a gold ring on layaway for someone special) wherein they have money invested in one option but a monetarily better "new” option is discovered. Participants are asked to rate on a 6-point scale whether they would stick with the less viable option that they had invested in or switch to the new, monetarily advantageous alternative. The scale ranges from “1” "most likely to choose” staying with the chosen option (e.g., continue paying at the old store [the sunk-cost option]) to "6" "most likely to choose" (buy from the new store [normatively correct option]) where higher scores are indicative of greater resistance to sunk cost.

\section{Results}

Because we hypothesized that glucose enrichment would lead to better and faster decisions, we performed separate one-tailed t-tests on participants' choices in each of the ADMC subsections as well as the overall decision score. We then performed similar analyses in each section for participant's response times. The A-DMC is standardized so that higher scores represent better decision making. Therefore, according to our hypothesis, glucose enriched subjects should have higher decision scores and faster response times compared to glucose deprived subjects. The means and standard deviations for each subsection and overall scores are presented in Table 1, response times are presented in Table 2. 
First we tested whether participants in the glucose condition made normatively better choices than participants in the placebo condition. The results of these analyses are presented in Table 3. As can be seen, glucose enrichment did not improve decision making in the simpler decision tasks. Decision making only improved in Applying Decision Rules, the subsection that involves more complex decision making.

Next we tested for effects of glucose on response time. The results of these analyses can be found in Table 4. For the overall A-DMC, glucose enriched participants responded significantly faster to decisions than participants in the glucose deprived condition. Similar glucose facilitation effects are depicted in the Recognizing Social Norms, Applying Decision Rules and Consistency in Risk Perception subsections. The subsections of Resistance to Framing, Under/overconfidence, and Resistance to Sunk Costs were not significantly influenced by the glucose manipulation. However, as can be seen in Tables 2 and 4, all of the means were in the predicted direction and the effect sizes were small but consistent.

\section{Discussion}

Glucose is an especially important factor to consider in decision making because of its ubiquity and variability. Our findings show that enriching blood glucose levels improves performance on complex decisions but has little to no effect on the quality of simpler decisions. This is consistent with prior research showing that glucose enhancement improves performance on complex cognitive tasks but not simpler ones (e.g., Scholey et al., 2001). The response time data served to operationalize processing speed and allowed us to measure an aspect of decision making that could otherwise be overlooked. We found evidence that glucose is an important factor for response time across most types of decision tasks. This finding is also consistent with 
research looking at the speed-facilitation effects of glucose and cognition (e.g., Benton et al., 1994).

These findings have many practical implications for future research and are especially relevant for technicians who make complex decisions that hinge on critically fast decision making. For example, consider air traffic controllers who must weigh multiple factors and make critical decisions quickly. Our study suggests that both optimality and response time for this type of decision will be significantly affected by blood-glucose level.

Finally, it should be noted that the glucose manipulation in this study was by no means extreme. Short fasting intervals and soft drinks are common in many diets. It may be that larger variations in glucose levels, such as those experienced by individuals with certain medical conditions, will have a greater influence on decision making. Nevertheless, the findings from our study add to a growing body of research that focuses on understanding how physiological and psychological factors interact to form the decision making process. 


\section{References}

Benton, D. (1990). The impact of increasing blood glucose on psychological functioning. Biological Psychology, 30, 13-19. doi:10.1016/0301-0511(90)90087-D

Benton D., Owens D.S., \& Parker, P.Y. (1994). Blood glucose memory and attention. Neuropsychologia, 32, 595- 607.

Benton, D., \& Nabb, S. (2003). Carbohydrate, memory, and mood. Nutrition Reviews, 61, 61-67.

Benton, D.. Owens, D. S., \& Parker, P. Y. (1994). Blood glucose influences memory and attention in young adults. Neuropsychologia, 32, 595-607.

Bos, M. W., Dijksterhuis, A., \& van Baaren, R. (2012). Food for thought? Trust your unconscious when energy is low. Journal of Neuroscience, Psychology, and Economics, 5, 124-130. doi:10.1037/a0027388

Bruine de Bruin, W., Parker, A. M., and Fischhoff, B. (2007). Individual differences in adult decision-making competence. Journal of Personality and Social Psychology, 92, 938-56.

Dickinson, D.J., McElroy, T. \& Stroh, N. (in press). The impact of glucose on Bayesian v. heuristic-based decision making. Journal of Neuroscience, Psychology, and Economics.

Donohoe, R. T., \& Benton, D. (1999). Cognitive functioning is susceptible to the level of blood glucose. Psychopharmacology, 145, 378-385.

Gonder-Frederick, L. A., Hall, J. L., Vogt, J. J., Cox, D. J., Green, J. J., \& Gold, P. E. (1987). Memory enhancement in elderly humans: Effects of glucose ingestion. Physiology \& Behavior, 41, 503-504. doi:10.1016/0031-9384(87)90087-4 
Kennedy, D. O., \& Scholey, A. B. (2000) Glucose administration, heart rate and cognitive performance: effects of increasing mental effort. Psychopharmacology, 149, 63-71.

Levin, I. P., Gaeth, G. J., Schreiber, J., \& Lauriola, M. (2002). A new look at framing effects: Distribution of effect sizes, individual differences, and independence of types of effects. Organizational Behavior and Human Decision Processes, 88, 411-429.

Levin, I. P., Schneider, S. L., \& Gaeth, G. J. (1998). All frames are not created equal: A typology and critical analysis of framing effects. Organizational Behavior and Human Decision Processes, 76, 149-188.

Masicampo, E. J., \& Baumeister, R. F. (2008). Toward a physiology of dual-process reasoning and judgment: Lemonade, willpower, and expensive rule-based analysis. Psychological Science, 19(3), 255-260.

McMahon, A. J., \& Scheel, M. H. (2010). Glucose promotes controlled processing: Matching, maximizing, and root beer. Judgment and Decision Making, 5(6), 450-457.

Messier, C., Pierre, J., Desrochers, A., Gravel, M., (1998). Dose-dependent action of glucose on memory processes in women: effect on serial position and recall priority. Cognitive Brain Research 7, 221-233.

Metzger, M.M., (2000). Glucose enhancement of a facial recognition task in young adults. Physiology and Behavior 68, 549-553.

Owens, D. S. \& Benton, D. (1994). The impact of raising blood glucose on reaction times. Neuropsychobiology, 30, 106-113.

Parker, A. M., \& Fischhoff, B. (2005). Decision-making competence: External validation through an individual differences approach. Journal of Behavioral Decision Making, $18,1-27$. 
Schächinger, H., Cox, D., Linder, L., Brody, S. \& Keller, U. (2003) Cognitive and psychomotor function in hypoglycemia: Response error patterns and retest reliability. Pharmacology, Biochemistry, and Behavior, 75, 915-920.

Scholey, A.B., Harper, S., \& Kennedy, D.O. (2001). Cognitive demand and blood glucose. Physiology \& Behavior 73, 585-592.

Simonson, I. (1989). Choice based on reasons: The case of attraction and compromise effects. Journal of Consumer Research, 16, 158-174.

Stanovich, K. E., \& West, R. F. (2000). Individual differences in reasoning: Implications for the rationality debate? The Behavioral and Brain Sciences, 23, 645-726.

Sunram-Lea, S. I., Foster, J.K., Durlach, P. \& Perez, C. (2001). Glucose facilitation of cognitive performance in healthy young adults: examination of the influence of fast-duration, time of day and pre-consumption plasma glucose levels. Psychopharmacology, 157, 46-54.

Sünram-Lea, S.I., Foster, J.K., Durlach, P. \& Perez, C. (2002). The effect of retrograde and anterograde glucose administration on memory performance in healthy young adults. Behavioural Brain Research 134, 505-516.

Wang, X.T., \& Dvorak, R.D. (2010). Sweet Future: Fluctuating Blood Glucose Levels Affect Future Discounting. Psychological Science, 21, 183-188. 


\section{Tables}

Table 1

Means and SD of Non-standardized A-DMC Scores

\begin{tabular}{lccccc}
\hline & \multicolumn{4}{c}{ Condition } & \multicolumn{3}{c}{ Glucose $^{\mathrm{a}}$} & & \multicolumn{2}{c}{ Placebo $^{\mathrm{a}}$} \\
\cline { 2 - 3 } \cline { 5 - 6 } A-DMC score & $M$ & $S D$ & & $M$ & $S D$ \\
\hline Resistance to Framing & 1.01 & 0.46 & & 1.02 & 0.54 \\
Recognizing Social Norms & 0.48 & 0.17 & & 0.44 & 0.20 \\
Under/overconfidence & 0.76 & 0.09 & & 0.78 & 0.09 \\
Applying Decision Rules & 0.76 & 0.13 & & 0.54 & 0.24 \\
Consistency in Risk Perception & 0.67 & 0.13 & & 0.67 & 0.09 \\
Resistance to Sunk Costs & 3.93 & 0.65 & & 4.00 & 0.49 \\
A-DMC Total & 1.22 & 0.13 & & 1.24 & 0.12 \\
\hline
\end{tabular}

Note. A-DMC Total is the average of the non-standardized component scores.

${ }^{\mathrm{a}} n=69$

Table 2

Means and SD of Response Times for A-DMC in seconds

\begin{tabular}{|c|c|c|c|c|}
\hline \multirow[b]{3}{*}{ A-DMC score } & \multicolumn{4}{|c|}{ Condition } \\
\hline & \multicolumn{2}{|c|}{ Glucose $^{\mathrm{a}}$} & \multicolumn{2}{|c|}{ Placebo $^{a}$} \\
\hline & $M$ & $S D$ & $M$ & $S D$ \\
\hline Resistance to Framing & 576.08 & 161.68 & 614.56 & 150.15 \\
\hline Recognizing Social Norms & 156.14 & 49.07 & 174.11 & 108.23 \\
\hline Under/overconfidence & 313.28 & 82.67 & 329.75 & 74.02 \\
\hline Applying Decision Rules & 313.28 & 69.25 & 375.53 & 102.70 \\
\hline Consistency in Risk Perception & 131.96 & 37.57 & 148.35 & 37.74 \\
\hline Resistance to Sunk Costs & 199.45 & 62.42 & 211.88 & 63.23 \\
\hline A-DMC Total & 283.71 & 69.25 & 309.03 & 66.88 \\
\hline
\end{tabular}

Note. A-DMC Total is the average of RTs (in seconds) for all component tasks.

${ }^{\mathrm{a}} n=69$ 
Table 3

Analysis of A-DMC Component Scores and Composite Score onetailed t-test

\begin{tabular}{lccr}
\hline A-DMC Component & $t(136)$ & $p$ & $d$ \\
\hline & & & \\
Resistance to Framing & -0.06 & 0.52 & -0.01 \\
Recognizing Social Norms & 1.12 & 0.13 & 0.19 \\
Under/overconfidence & -1.26 & 0.89 & -0.22 \\
Applying Decision Rules & 1.70 & 0.05 & 0.29 \\
Consistency in Risk Perception & 0.18 & 0.42 & 0.03 \\
Resistance to Sunk Costs & -0.62 & 0.73 & -0.10 \\
A-DMC Total & -0.99 & 0.69 & -0.17 \\
\hline
\end{tabular}

Note. The subsections containing a negative $t$ value were not statistically significant in the opposite direction for either the one-tailed or two-tailed test.

Table 4

Analysis of Response Times for A-DMC one-tailed t-test

\begin{tabular}{lccc}
\hline A-DMC Component & $t(136)$ & $p$ & $d$ \\
\hline & & & \\
Resistance to Framing & 1.42 & 0.08 & 0.24 \\
Recognizing Social Norms & 2.29 & 0.01 & 0.39 \\
Under/overconfidence & 1.23 & 0.11 & 0.21 \\
Applying Decision Rules & 2.93 & 0.00 & 0.50 \\
Consistency in Risk Perception & 2.56 & 0.00 & 0.44 \\
Resistance to Sunk Costs & 1.16 & 0.12 & 0.20 \\
A-DMC Total & 2.24 & 0.02 & 0.38 \\
\hline
\end{tabular}

